\title{
Association of GCKR Gene Polymorphisms with the Risk of Nonalcoholic Fatty Liver Disease and Coronary Artery Disease in a Chinese Northern Han Population
}

\author{
Hui Gao ${ }^{\# 1}$, Shousheng Liü2,4, Zhenzhen Zhao ${ }^{2,4}$, Xinjuan Yu ${ }^{2}$, Qun Liư ${ }^{1}$, Yongning Xin*1,3,4 \\ and Shiying Xuan*1,3,4
}

${ }^{1}$ Department of Infectious Disease, Qingdao Municipal Hospital, Qingdao University, Qingdao, Shandong, China; ${ }^{2}$ Central Laboratories, Qingdao Municipal Hospital, Qingdao University, Qingdao, Shandong, China; ${ }^{3}$ Department of Gastroenterology, Qingdao Municipal Hospital, Qingdao University, Qingdao, Shandong, China; ${ }^{4}$ Digestive Disease Key Laboratory of Qingdao,

Qingdao, Shandong, China

\begin{abstract}
Background and Aims: Accumulated studies have evaluated the effects of glucokinase regulatory protein (GCKR) gene polymorphisms on the risk of nonalcoholic fatty liver disease (NAFLD) and coronary artery disease (CAD), but the association of GCKR polymorphisms with the risk of NAFLD and $C A D$ in the Chinese Han population have remained unclear. The aim of this study was to investigate the association between GCKR gene polymorphisms (rs780094 and rs1260326) and the risk of NAFLD and CAD in NAFLD patients in a Chinese Northern Han population. Methods: GCKR rs780094 and rs1260326 gene polymorphisms were genotyped by polymerase chain reaction sequencing for B-type ultrasonography-proven NAFLD patients with $(n=82)$ or without $(n=142)$ CAD, and in healthy controls $(n=152)$. Serum lipid profiles' levels were determined using biochemical methods. Statistical analyses were conducted using SPSS 22.0 statistical software. Results: As the results showed, significant differences in the serum lipid profiles existed between each group. No significant differences were observed in the distributions of genotypes and alleles of GCKR rs780094 and rs1260326 in each group. The GCKR rs780094 $\mathrm{T}$ and rs1260326 T allele carriers possessed decreased body mass index value, and serum fasting plasma glucose and TG levels in the overall subjects, respectively. In addition, the GCKR rs780094 Tallele carriers possessed decreased serum fasting plasma glucose level in the controls and NAFLD + CAD
\end{abstract}

Keywords: Non-alcoholic fatty liver disease; Glucokinase regulatory protein gene; Polymorphism; Coronary artery disease.

Abbreviations: ALP, alkaline phosphatase; ALT, alanine aminotransferase; AST, aspartate aminotransferase; BMI, body mass index; BIL, bilirubin; CAD, coronary artery disease; CI, confidence intervals; FPG, fasting plasma glucose; GCK, glucokinase; GCKR, glucokinase regulatory protein; GWAS, genome-wide association studies; HDL, high-density lipoprotein; LDL, low-density lipoprotein; NAFLD, nonalcoholic fatty liver disease; $O R$, odds ratio; $P C R$, polymerase chain reaction; r-GT, glutamyltransferase; SD, standard deviation; SNP, single nucleotide polymorphism; TC, total cholesterol; TG, triglyceride.

Received: 22 July 2019; Revised: 7 October 2019; Accepted: 8 December 2019 ${ }^{\#}$ Authors contributed equally to this work.

*Correspondence to: Yongning Xin, Department of Infectious Disease, Qingdao Municipal Hospital, Qingdao University, 1 Jiaozhou Road, Qingdao, Shandong 266011, China. Tel: +86-532-82789463, Fax: +86-532-85968434, E-mail: xinyongning@163.com; Shiying Xuan, Department of Gastroenterology, Qingdao Municipal Hospital, Qingdao University, 1 Jiaozhou Road, Qingdao, Shandong 266011, China. Tel: +86-532-88905508, Fax: +86-532-88905293, E-mail: xuansydxy@163.com patients. Conclusions: GCKR rs780094 and rs1260326 polymorphisms were found to be not associated with the risk of NAFLD nor of CAD in NAFLD patients in this Chinese Northern Han population. GCKR rs780094 T and rs1260326 T alleles could affect the body mass index value and serum fasting plasma glucose and triglyceride levels.

Citation of this article: Gao H, Liu S, Zhao Z, Yu X, Liu Q, Xin $Y$, et al. Association of GCKR gene polymorphisms with the risk of nonalcoholic fatty liver disease and coronary artery disease in a Chinese Northern Han population. J Clin Transl Hepatol 2019;7(4):297-303. doi: 10.14218/JCTH.2019.00030.

\section{Introduction}

Nonalcoholic fatty liver disease (NAFLD) is the most prevalent chronic liver disease in the world and is regarded as a severe public health concern. ${ }^{1}$ The overall prevalence of NAFLD is approximately $25 \%$ worldwide and is $23 \%, 31 \%$ and $27 \%$ in Europe, South America and Asia, respectively. ${ }^{2-4}$ Many factors contribute to the development of NAFLD, such as aging, hyperlipidemia, insulin resistance, type 2 diabetes, diet, genetics and so on. ${ }^{5-8}$ Accumulated lines of evidence have suggested that coronary artery disease (CAD) plays an important role in the progression of NAFLD and is associated with the severity of NAFLD.

CAD has become the most important cause of mortality among NAFLD patients. ${ }^{9}$ Although some defects of liver biopsy exist, such as invasive, sample errors and operator dependence, it remains the gold standard for diagnosis of NAFLD. ${ }^{10,11}$ In recent years, some noninvasive diagnostic methods have been developed and their effects have been tested in different countries and populations. Genome-wide association studies (GWAS) have identified several important single nucleotide polymorphism (SNP) sites which are tightly associated with the risk of development of NAFLD; these include PNPLA3 rs738409, TM6SF2 rs58542926, and LYPLAL1 rs $12137855 .{ }^{12}$ In consideration of the differences of genetic background of NAFLD patients, it is necessary to identify the relationship of gene polymorphisms with the risk of NAFLD in different countries and races.

Glucokinase (GCK) is a phosphorylating enzyme which can regulate hepatic glucose metabolism and activate hepatic lipogenesis. ${ }^{13}$ The GCKR gene encodes the glucokinase 
regulatory protein (GCKRP), which can bind to GCK allosterically and regulate the activity of GCK. ${ }^{14}$ In 2011, Speliotes et al. ${ }^{15}$ identified the GCKR rs780094 by GWAS as a significant SNP site associated with the risk of NAFLD. Subsequent studies in different districts reported controversial conclusions of GCKR rs780094 in NAFLD. The GCKR rs780094 T allele was found to be tightly associated with susceptibility to NAFLD in Americans and Malaysians, acting as a risk factor for NAFLD. ${ }^{15,16}$ However, no significant association between GCKR rs780094 and the risk of NAFLD was observed in the community-based study in Suzhou and Shanghai, and in obese adolescents in Beijing, China. ${ }^{17-20}$ On the contrary, Lin et al. ${ }^{21}$ reported that GCKR rs780094 was associated with an increased risk of NAFLD in obese children in Taiwan. ${ }^{22}$ Therefore, large-scale population-based studies in different districts are needed to investigate the effect of GCKR rs780094 on the development of NAFLD in China.

In addition, GWAS analyses conducted in Finnish, Swedish and Danish populations found a strong linkage disequilibrium of GCKR rs780094 with GCKR rs1260326. ${ }^{23,24}$ In Japan, Kawaguchi et al. ${ }^{25}$ found that GCKR rs1260326 was a significant risk factor for the development of NAFLD. In France, Petit et al. ${ }^{26}$ reported that GCKR rs1260326 influences the liver fat content in patients with type 2 diabetes. Di et al. ${ }^{27}$ reported that GCKR rs1260326 could increase the liver fat content, although the detailed role of GCKR rs1260326 in NAFLD remains controversial. Besides, Simons et al. ${ }^{28}$ conducted a meta-analysis to investigate the relationship of GCKR polymorphisms with the risk of CAD, and a tight association of $G C K R$ variants with the risk of CAD was observed.

In consideration of the unclear effects of GCKR rs780094 and rs1260326 on NAFLD in Chinese, and the tight association of CAD and NAFLD, it is of interest to explore the effects of GCKR rs780094 and rs1260326 on the development of NAFLD and the development of CAD in NAFLD patients from this population.

The aim of this study was to explore the relationship between GCKR gene polymorphisms (rs780094 and rs1260326) with the risk of NAFLD, the risk of CAD in NAFLD patients in a Chinese Northern Han population, and the effects of GCKR rs780094 and rs1260326 on the levels of serum lipid profiles.

\section{Methods}

\section{Subjects}

This case-control study was conducted according to the principles of the Declaration of Helsinki and its appendices. ${ }^{29}$ This study was approved by the ethics committee of Qingdao Municipal Hospital (Qingdao, China). All the subjects included in this study were of the Chinese Northern Han population, and each signed a written informed consent form.

From June 2018 to December 2018, 225 unrelated adult Chinese Northern Han patients with NAFLD, of both genders, that were diagnosed by B-type ultrasonography were recruited. Among these NAFLD patients, 82 suffered from CAD (41 males, 41 females; mean age of $55.62 \pm 7.187$ years) and 142 did not have CAD ( 75 males, 67 females; mean age of $50.14 \pm 11.842$ years). A total of 152 healthy controls, matched for sex and age ( 75 males, 77 females; mean age of $51.38 \pm 13.276$ years) were included. All the patients were recruited from the Departments of Gastroenterology and Cardiology and the Health Examination Center of Qingdao Municipal Hospital.
Standard clinical evaluations of the NAFLD patients were performed according to the criteria of American Association for the Study of Liver Diseases. ${ }^{21}$ Subjects with the following symptoms were excluded from the NAFLD cohort: 1) excessive alcohol intake (males $>210 \mathrm{~g} / \mathrm{w}$, females $>140 \mathrm{~g} / \mathrm{w}$ ); 2) viral hepatitis, such as hepatitis B or hepatitis $C$; 3 ) druginduced hepatitis; 4) other endocrine and metabolic disorders, such as renal disease. CAD was diagnosed using the findings from percutaneous coronary angiogram conducted by two experienced interventional cardiologists, and defined as the presence of at least $50 \%$ stenosis in at least one of the coronary arteries. The healthy controls were also subjected to the same diagnosis routines for NAFLD and CAD, as well as the laboratory and general examinations.

\section{Biochemical analyses}

The basic clinical pathological information (gender, age, height, and weight) was obtained by a standard study questionnaire. The body mass index (BMI) was calculated as mass weight $(\mathrm{kg}) /$ height $^{2}\left(\mathrm{~m}^{2}\right)$. All the subjects underwent a $12-h$ overnight fast, and then the blood samples were collected into ethylene diaminetetraacetic acid-containing tubes. Serum fasting plasma glucose (FPG), total cholesterol (TC), triglycerides (TG), high-density lipoprotein (HDL), lowdensity lipoprotein (LDL), alanine aminotransferase (ALT), aspartate aminotransferase (AST), glutamyltransferase $(r-G T)$, alkaline phosphatase (ALP), and bilirubin (BIL) were measured using standard clinical laboratory techniques. ${ }^{30}$

\section{Genomic DNA extraction and genotyping}

Genomic DNA was extracted and stored as previously described. $^{31}$ The primers for PCR amplification containing rs780094 were 5'-ACGTTGGATGAAGTGGTGGGATTACAGGAG-3' and 5'-ACGTTGGATGAGGGCCCCAGTITITAGAC-3', rs1260326 5'-ACGTTGGATGACCTGGGTCCCTTTGTCAC-3' and '5'-ACGTTGGATGAGAAGACCAACCACATCCAG-3' and were synthesized by Beijing BoMiao Biotech Company (China). The PCR amplification profile was as follows: predenaturation at $94^{\circ} \mathrm{C}$ for $5 \mathrm{~m}$, followed by 40 cycles of denaturation at $94^{\circ} \mathrm{C}$ for $20 \mathrm{~s}$, annealing at $56^{\circ} \mathrm{C}$ for $30 \mathrm{~s}$, extending at $72^{\circ} \mathrm{C}$ for $60 \mathrm{~s}$, followed by the final extension at $72^{\circ} \mathrm{C}$ for $3 \mathrm{~m}$. The target amplified fragments were detected by $2 \%$ gel electrophoresis, with the predicted fragment length. The genotypes of rs780094 and rs1260326 were detected by direct DNA sequencing using the Applied Biosystems Inc. (USA) Veriti-384 Prism Sequence Detection System, and the raw data were analyzed using MassARRAY TYPER4.0 software (Agena Bioscience, USA). Genotyping was performed in a blinded fashion and the success rates were $>95 \%$.

\section{Statistical analysis}

Statistical analysis was conducted using the SPSS 22.0 statistical software (SPSS Inc., USA). Student's $t$-test or $\chi^{2}$ test were used to analyzed the differences in characteristics of different groups. First, continuous variables were tested by normal distribution and $\mathrm{F}$ test; if yes, the baseline characteristics were expressed as the mean \pm standard deviation (SD), and if no, the baseline characteristics were expressed as the median \pm quartile. Hardy-Weinberg equilibrium was between expected and observed genotype distributions and was estimated using the $\chi^{2}$ test. Genotype and allele frequencies were 
Gao H. et al: GCKR with NAFLD and CAD

Table 1. Baseline characteristics of the study participants ${ }^{\mathrm{a}}$

\begin{tabular}{|c|c|c|c|c|c|c|c|}
\hline Characteristic & Controls & NAFLD & NAFLD + CAD & $p$ & $p_{1}$ & $p_{2}$ & $p_{3}$ \\
\hline Age $(y)$ & $51.38 \pm 13.276$ & $50.14 \pm 11.842$ & $55.62 \pm 7.187$ & 0.156 & - & - & - \\
\hline Gender (F/M) & $77 / 75$ & $67 / 75$ & $41 / 41$ & 0.174 & - & - & - \\
\hline BMI $\left(\mathrm{kg} / \mathrm{m}^{2}\right)$ & $23.45 \pm 3.376$ & $26.74 \pm 2.805$ & $25.24 \pm 2.606$ & $<0.001$ & $<0.001$ & $<0.001$ & 0.003 \\
\hline FPG $(\mathrm{mmol} / \mathrm{L})$ & $4.80 \pm 1.479$ & $5.00 \pm 1.343$ & $5.90 \pm 2.180$ & $<0.001$ & 0.010 & $<0.001$ & 0.001 \\
\hline $\mathrm{TC}(\mathrm{mmol} / \mathrm{L})$ & $4.99 \pm 1.192$ & $5.48 \pm 0.822$ & $4.56 \pm 1.274$ & $<0.001$ & 0.010 & $<0.001$ & $<0.001$ \\
\hline TG (mmol/L) & $1.45 \pm 1.009$ & $1.90 \pm 1.903$ & $2.23 \pm 4.396$ & $<0.001$ & 0.003 & $<0.001$ & 1.000 \\
\hline $\mathrm{HDL}(\mathrm{mmol} / \mathrm{L})$ & $1.28 \pm 0.387$ & $1.24 \pm 0.198$ & $1.05 \pm 0.294$ & $<0.001$ & 1.000 & $<0.001$ & $<0.001$ \\
\hline LDL (mmol/L) & $3.10 \pm 0.708$ & $3.27 \pm 0.555$ & $2.75 \pm 0.972$ & $<0.001$ & 0.034 & 0.006 & $<0.001$ \\
\hline ALT (U/L) & $19.90 \pm 11.155$ & $34.40 \pm 26.165$ & $28.90 \pm 25.044$ & $<0.001$ & $<0.001$ & 0.006 & 0.019 \\
\hline AST (U/L) & $21.93 \pm 8.877$ & $24.16 \pm 8.994$ & $44.67 \pm 69.649$ & 0.067 & - & - & - \\
\hline $\mathrm{r}-\mathrm{GT}(\mathrm{mmol} / \mathrm{L})$ & $26.30 \pm 24.367$ & $39.63 \pm 26.481$ & $47.89 \pm 85.986$ & $<0.001$ & $<0.001$ & 0.010 & 0.019 \\
\hline ALP (mmol/L) & $75.14 \pm 24.661$ & $71.62 \pm 18.272$ & $85.79 \pm 21.840$ & $<0.001$ & 0.852 & $<0.001$ & $<0.001$ \\
\hline $\mathrm{BIL}(\mathrm{mmol} / \mathrm{L})$ & $13.20 \pm 5.358$ & $13.30 \pm 4.957$ & $14.44 \pm 7.020$ & 0.475 & - & - & - \\
\hline
\end{tabular}

Abbreviations: NAFLD, patients with nonalcoholic fatty liver disease; NAFLD + CAD, patients with nonalcoholic fatty liver disease and coronary artery disease; BMI, body mass index; FPG, fasting plasma glucose; TC, total cholesterol; TG, triglycerides; HDL, high-density lipoprotein; LDL, low-density lipoprotein; ALT, alanine aminotransferase; AST, aspartate aminotransferase; r-GT, glutamyltransferase; ALP, alkaline phosphatase; BIL, bilirubin.

$p$, controls vs. NAFLD vs. NAFLD + CAD ; $p_{1}$, NAFLD vs. controls; $p_{2}$, NAFLD + CAD vs. controls; $p_{3}$, NAFLD + CAD vs. NAFLD.

${ }^{\mathrm{a}}$ Values are expressed as median \pm quartile.

assessed by counting the DNA sequencing data, and the distributions in NAFLD versus controls were analyzed by Pearson's $\chi^{2}$ test or Fisher's exact test, where appropriate. Association between polymorphisms and NAFLD/CAD were analyzed by logistic regression and odds ratios (ORs) with $95 \%$ confidence intervals (CIs). A $p$-value $<0.05$ was considered statistically significant.

\section{Results}

\section{Characteristics of the study participants}

The baseline characteristics of the three groups (NAFLD, NAFLD with CAD, and healthy controls) according to the experimental requirements are shown in Table 1 . There were no significant differences in age or gender among the three groups (all $p>0.05$ ). The increased serum levels of BMI, TC, TG, LDL, ALT, AST, and r-GT were observed in the NAFLD patients compared to the healthy controls (all $p<$ 0.05). In the NAFLD + CAD group, the BMI value and serum levels of FPG, TC, TG, LDL, ALT, AST, r-GT, and ALP were higher and the serum HDL level was lower than in healthy controls (all $p<0.05$ ). Compared to the NAFLD patients, the patients with NAFLD and CAD possessed higher serum levels of FPG, AST and ALP, and lower BMI value and serum levels of TC, HDL, and LDL (all $p<0.05$ ).

\section{GCKR rs780094 and rs1260326 genotype and allele distribution}

The genotype distributions of GCKR rs780094 and rs1260326 were in accordance with the Hardy-Weinberg equilibrium in each group (all $p>0.05$ ) (Table 2 ). As the results show in Table 3, there were no significant differences in the genotypic and allelic distributions of GCKR rs780094 and rs1260326 between the NAFLD patients and healthy controls, NAFLD + CAD patients and healthy controls, nor NAFLD + CAD patients and NAFLD patients, respectively (all $p>0.05$ ). These results suggested that GCKR rs780094 and rs1260326 polymorphisms did not associate with the risk of NAFLD in this Northern Chinese Han population, and GCKR rs780094 and rs1260326 polymorphisms did not increase the risk of CAD in these NAFLD patients.

\section{Association of GCKR polymorphisms with clinical parameters in each group}

The effects of GCKR rs780094 T or rs1260326 Tallele on the clinical characteristics of NAFLD patients, NAFLD + CAD patients, and overall series were analyzed. As the results show in Table 4 and Table 5, the BMI value and serum FPG and TG levels were significantly lower in the rs780094 T and rs1260326 T allele carriers than in the non-carriers in the overall series (all $p<0.05$ ), respectively. In addition, the serum level of FPG in rs780094 T carriers was higher than in non-carriers in the NAFLD + CAD group $(p<0.05)$. No marked difference of other clinical parameters was observed between the carriers and non-carriers of the rs780094 T or rs1260326 T allele in each group $(p>0.05)$. Multivariate

Table 2. Results of the Hardy-Weinberg equilibrium test ${ }^{\mathrm{a}}$

\begin{tabular}{llllll}
\hline Gene locus groups & CC & CT & $T$ & $\chi^{2}$ & $p$ value \\
\hline rs780094 & & & & & \\
$\quad$ Controls & 26 & 81 & 45 & 1.040 & 0.308 \\
$\quad$ NAFLD & 26 & 71 & 45 & 0.047 & 0.828 \\
$\quad$ NAFLD + CAD & 18 & 39 & 25 & 0.014 & 0.708 \\
rs1260326 & & & & & \\
$\quad$ Controls & 23 & 82 & 46 & 1.890 & 0.169 \\
$\quad$ NAFLD & 24 & 73 & 45 & 0.372 & 0.542 \\
$\quad$ NAFLD + CAD & 15 & 41 & 26 & 0.028 & 0.868 \\
\hline
\end{tabular}

Abbreviations: NAFLD, patients with nonalcoholic fatty liver disease; NAFLD + $C A D$, patients with nonalcoholic fatty liver disease and coronary artery disease. ${ }^{\mathrm{a}}$ Data were compared by chi-square test. 
Gao H. et al: GCKR with NAFLD and CAD

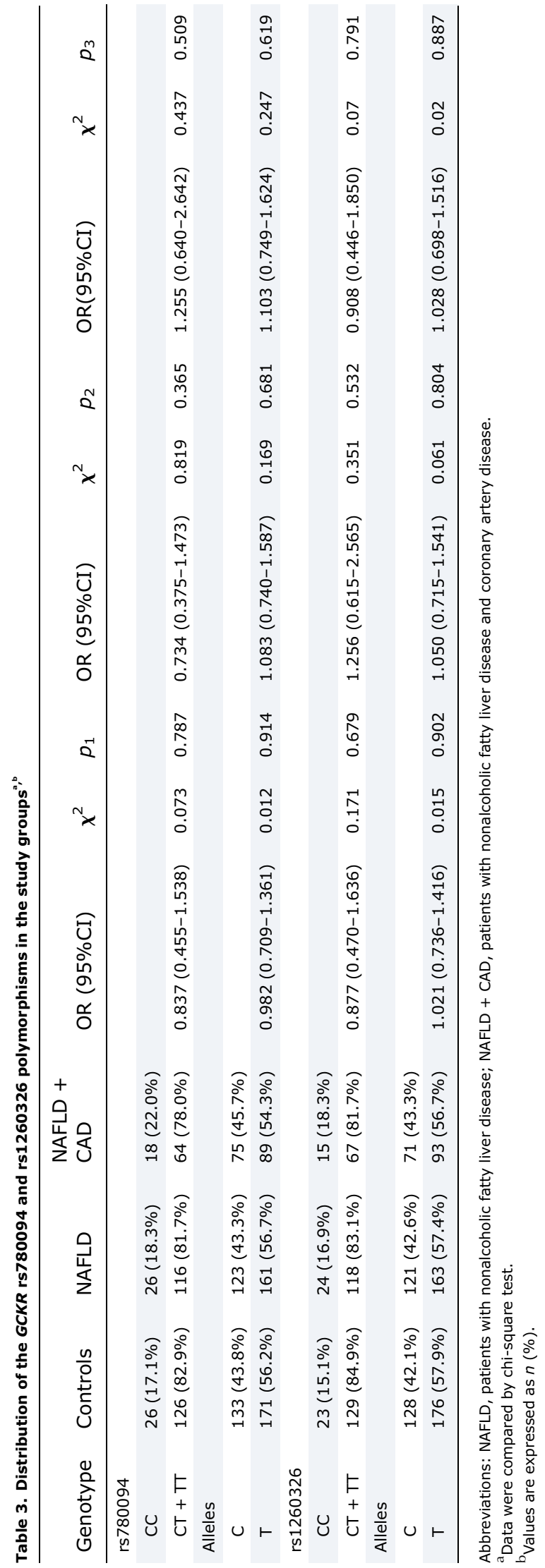

logistic regression analysis showed that GCKR rs780094 is the dependent factor associated with FPG $(p<0.05)$.

\section{Discussion}

NAFLD can be caused by many risk factors, such as diet, genetics and so on. Genetic susceptibility genes are a type of risk factor that can contribute to the development of NAFLD. In this study, we investigated the relationship between GCKR rs780094 and rs1260326 gene polymorphisms and the risk of NAFLD in a general Chinese Northern Han population, and the risk of CAD in NAFLD patients in this population for the first time. Our results showed that the genotype distributions of GCKR rs780094 and rs1260326 were not associated with the risk of NAFLD, and not associated with the risk of CAD in NAFLD patients in this Chinese Northern Han population. In addition to the BMI value and serum FPG and TG levels, the Tallele of GCKR rs780094 and rs1260326 also did not significantly affect the clinical parameters in NAFLD patients and NAFLD + CAD patients. These data suggest that GCKR rs780094 and rs1260326 polymorphisms are not associated with the risk of NAFLD in the general Chinese Northern Han population, and GCKR rs780094 and rs1260326 polymorphisms do not increase the risk of CAD in NAFLD patients in the Chinese Northern Han population.

Accumulated studies have explored the effects of GCKR polymorphisms on the risk of NAFLD but the results have been inconsistent. ${ }^{16-18,32}$ Some previous studies and our study suggested that GCKR rs780094 and rs1260326 polymorphisms are not associated with the risk of NAFLD, the probable reason for this finding may be due to the ethnic differences among the NAFLD patients studied. The relationship between GCKR polymorphism and NAFLD or nonalcoholic steatohepatitis may be modified by the change and confluence of race, therefore the effects of a GCKR polymorphism may become more dominant and significant in European populations than they are in Asian populations. Therefore, the GCKR rs780094 T allele might increase the risk of NAFLD in European populations but not in Asian populations. ${ }^{15,17,18,33}$ A higher prevalence of NAFLD has been observed in some Indians that possess European ancestry, as opposed to those that possess Asian ancestry, which might explain the above hypothesis. ${ }^{34}$

The GCKR gene encodes GCKRP, an enzyme that plays a regulatory role in hepatic GCK activity. ${ }^{35,36}$ The GCKR rs1260326 functional variant P446L increases GCK activity by down-regulating the level of fructose 6-phosphate. ${ }^{37}$ Increased GCK activity is tightly accompanied by elevated hepatic glycolytic flux, de novo lipogenesis, and hepatic TG level. ${ }^{13,38}$ In this study, our results showed that the GCKR rs780094 and rs1260326 T allele were significantly associated with decreasing serum FPG level among the overall patients and the NAFLD + CAD patients. These results suggest that GCKR rs780094 and rs1260326 may repress the activity of GCKRP to decrease the serum FPG level.

Previous studies also investigated the association of GCKR polymorphisms with the risk of CAD. In a dietary intervention study, Shen et al. ${ }^{39}$ found that the GCKR rs1260326 T allele may increase the risk of atherosclerosis. Lian et al. ${ }^{40}$ demonstrated the positive association of GCKR rs780093 with the risk of CAD in an older Chinese Han population, through a case-control study. On the contrary, Járomi et al. ${ }^{41}$ did not detect any association of GCKR rs1260326 with the susceptibility of stroke. Bi et al. ${ }^{42}$ also found that the GCKR rs780094 


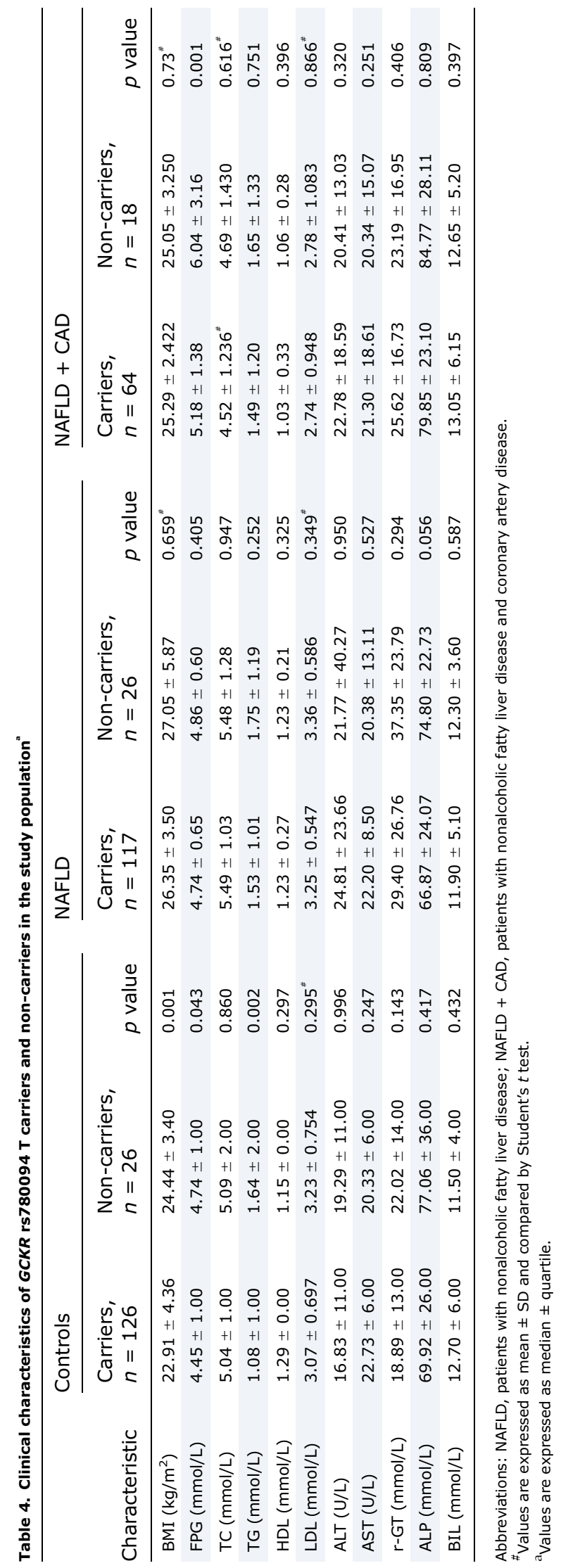

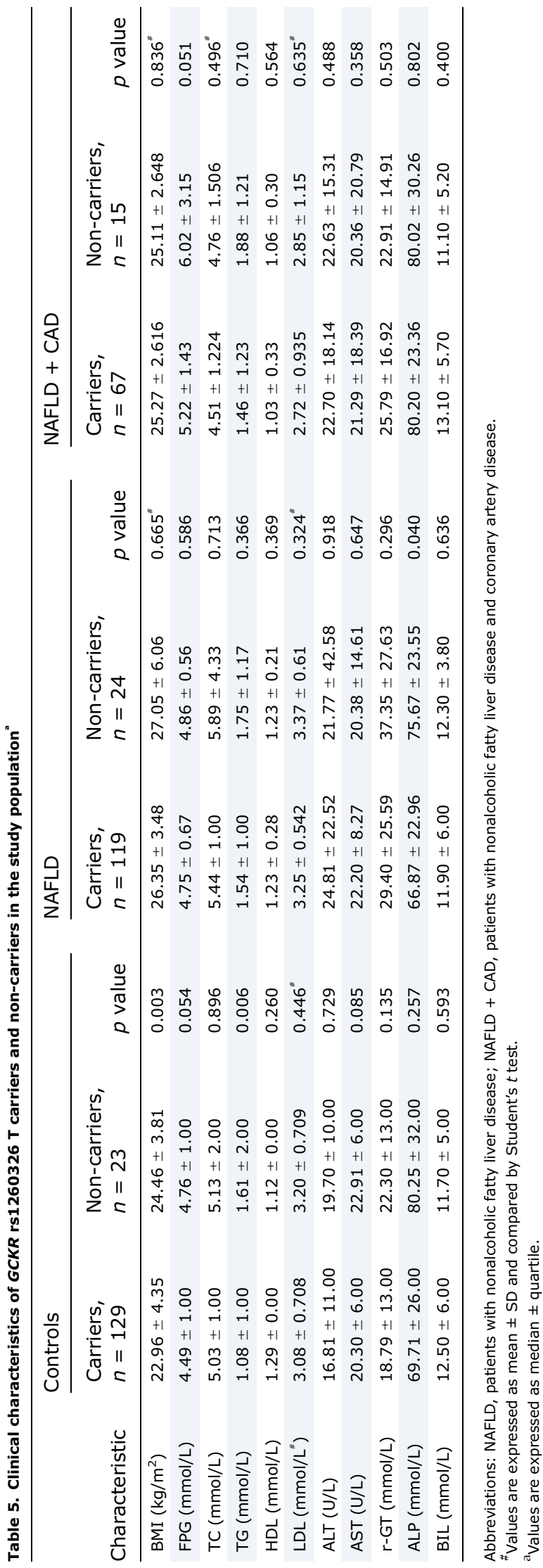


SNP had no significant association with the incidence of CAD or stroke. SNPs of GCKR rs780094 showed strong linkage disequilibrium with rs1260326. ${ }^{24,39,40}$ Our results showed that GCKR rs780094 and rs1260326 genotype distributions were not associated with the occurrence of CAD in NAFLD patients but that the rs780094 $\mathrm{T}$ allele could decrease the serum FPG levels in the NAFLD + CAD group. These results indicated that GCKR rs780094 and rs1260326 were not the risk factor of CAD in NAFLD patients but that the rs780094 T allele could affect serum FPG level in the NAFLD + CAD patients of our Chinese Northern Han population.

Some limitations of our study should be acknowledged. First, all the subjects in this study were of the Chinese Northern Han population, so that the applicability of our conclusion to other ethnic populations requires further study for confirmation. Second, ultrasonography was used to diagnose NAFLD because of the difficulty in conducting liver biopsy. Third, our study did not grade the severity of NAFLD patients. Finally, a greater number of subjects should be included in the further studies to verify this conclusion in the Chinese Han population and other ethnic populations.

\section{Conclusions}

In summary, we investigated the relationship of GCKR rs780094 and rs1260326 gene polymorphisms with the risk of NAFLD as well as the risk of CAD in NAFLD patients in the Chinese Northern Han population. We concluded that GCKR rs780094 and rs1260326 gene polymorphisms did not associate with the risk of NAFLD nor with the risk of CAD in NAFLD patients in the Chinese Northern Han population. In addition, the GCKR rs780094 T and rs1260326 T alleles did affect the $B M I$ value and serum FPG and TG levels in the overall subjects and NAFLD + CAD patients.

\section{Acknowledgments}

This study was supported by grants from the National Natural Science Foundation of China (31770837) and the Qingdao People's Livelihood Science and Technology Plan (18-6-1-68-nsh).

\section{Conflict of interest}

The authors have no conflict of interests related to this publication.

\section{Author contributions}

Study concept and design ( $Y X$ and $S X$ ), acquisition of the data (HG, SL, ZZ, XY, and QL), analysis and interpretation of the data (HG, SL, and $Z Z$ ), drafting of the manuscript (HG and $\mathrm{SL})$, critical revision of the manuscript for important intellectual content (YX and SX) supervision (YX and SX). All the authors read and approved the final manuscript.

\section{References}

[1] Danford CJ, Yao ZM, Jiang ZG. Non-alcoholic fatty liver disease: a narrative review of genetics. J Biomed Res 2018;32:389-400. doi: 10.7555/JBR.32. 20180045.

[2] Younossi Z, Anstee QM, Marietti M, Hardy T, Henry L, Eslam M, et al. Global burden of NAFLD and NASH: trends, predictions, risk factors and prevention. Nat Rev Gastroenterol Hepatol 2018;15:11-20. doi: 10.1038/nrgastro. 2017.109.
[3] Ibrahim SH, Hirsova P, Gores GJ. Non-alcoholic steatohepatitis pathogenesis: sublethal hepatocyte injury as a driver of liver inflammation. Gut 2018;67: 963-972. doi: 10.1136/gutjnl-2017-315691.

[4] Fiorucci S, Biagioli M, Distrutti E. Future trends in the treatment of non-alcoholic steatohepatitis. Pharmacol Res 2018;134:289-298. doi: 10.1016/j. phrs.2018.07.014.

[5] Younossi ZM, Stepanova M, Negro F, Hallaji S, Younossi Y, Lam B, et al. Nonalcoholic fatty liver disease in lean individuals in the United States. Medicine (Baltimore) 2012;91:319-327. doi: 10.1097/MD.0b013e3182779d49.

[6] Liangpunsakul S, Chalasani N. Lipid mediators of liver injury in nonalcoholic fatty liver disease. Am J Physiol Gastrointest Liver Physiol 2019;316:G75G81. doi: 10.1152/ajpgi.00170.2018.

[7] Feng S, Dai Z, Liu AB, Huang J, Narsipur N, Guo G, et al. Intake of stigmasterol and $\beta$-sitosterol alters lipid metabolism and alleviates NAFLD in mice fed a high-fat western-style diet. Biochim Biophys Acta Mol Cell Biol Lipids 2018; 1863:1274-1284. doi: 10.1016/j.bbalip.2018.08.004.

[8] Anstee QM, Day CP. The genetics of NAFLD. Nat Rev Gastroenterol Hepatol 2013;10:645-655. doi: 10.1038/nrgastro.2013.182.

[9] Vilar CP, Cotrim HP, Florentino GS, Bragagnoli G, Schwingel PA, Barreto CP. Nonalcoholic fatty liver disease in patients with coronary disease from a Brazil northeast area. Arq Gastroenterol 2015;52:111-116. doi: 10 . 1590/S0004-28032015000200007.

[10] Krishan S, Jain D, Bathina Y, Kale A, Saraf N, Saigal S, et al. Non-invasive quantification of hepatic steatosis in living, related liver donors using dualecho Dixon imaging and single-voxel proton spectroscopy. Clin Radiol 2016; 71:58-63. doi: 10.1016/j.crad.2015.10.002.

[11] Zhang Q, Zhang HM, Qi WQ, Zhang YG, Zhao P, Jiao J, et al. 3.0T ${ }^{1} \mathrm{H}$ magnetic resonance spectroscopy for assessment of steatosis in patients with chronic hepatitis C. World J Gastroenterol 2015;21:6736-6744. doi: 10.3748/wjg. v21.i21.6736.

[12] León-Mimila P, Vega-Badillo J, Gutiérrez-Vidal R, Villamil-Ramírez H, Villareal-Molina T, Larrieta-Carrasco $\mathrm{E}$, et al. A genetic risk score is associated with hepatic triglyceride content and non-alcoholic steatohepatitis in Mexicans with morbid obesity. Exp Mol Pathol 2015;98:178-183. doi: 10.1016/j. yexmp.2015.01.012.

[13] Peter A, Stefan N, Cegan A, Walenta M, Wagner S, Königsrainer A, et al. Hepatic glucokinase expression is associated with lipogenesis and fatty liver in humans. J Clin Endocrinol Metab 2011;96:E1126-E1130. doi: 10. 1210/jc.2010-2017.

[14] Iynedjian PB. Molecular physiology of mammalian glucokinase. Cell Mol Life Sci 2009;66:27-42. doi: 10.1007/s00018-008-8322-9.

[15] Speliotes EK, Yerges-Armstrong LM, Wu J, Hernaez R, Kim LJ, Palmer CD, et al. Genome-wide association analysis identifies variants associated with nonalcoholic fatty liver disease that have distinct effects on metabolic traits. PLoS Genet 2011;7:e1001324. doi: 10.1371/journal.pgen.1001324.

[16] Tan HL, Zain SM, Mohamed R, Rampal S, Chin KF, Basu RC, et al. Association of glucokinase regulatory gene polymorphisms with risk and severity of nonalcoholic fatty liver disease: an interaction study with adiponutrin gene. J Gastroenterol 2014;49:1056-1064. doi: 10.1007/s00535-013-0850-x.

[17] Yang H, Chen G, Song C, Li D, Ma Q, Chen G, et al. A novel index including SNPs for the screening of nonalcoholic fatty liver disease among elder Chinese: A population-based study. Medicine (Baltimore) 2018;97:e0272. doi: 10.1097/MD.0000000000010272.

[18] Wang X, Liu Z, Wang K, Wang Z, Sun X, Zhong L, et al. Additive effects of the risk alleles of PNPLA3 and TM6SF2 on non-alcoholic fatty liver disease (NAFLD) in a Chinese population. Front Genet 2016;7:140. doi: 10. 3389/fgene. 2016.00140 .

[19] Yang Z, Wen J, Tao X, Lu B, Du Y, Wang M, et al. Genetic variation in the GCKR gene is associated with non-alcoholic fatty liver disease in Chinese people. Mol Biol Rep 2011;38:1145-1150. doi: 10.1007/s11033-010-0212-1.

[20] Shang XR, Song JY, Liu FH, Ma J, Wang HJ. GWAS-identified common variants with nonalcoholic fatty liver disease in Chinese children. J Pediatr Gastroenterol Nutr 2015;60:669-674. doi: 10.1097/MPG.0000000000000662.

[21] Lin YC, Chang PF, Chang MH, Ni YH. Genetic variants in GCKR and PNPLA3 confer susceptibility to nonalcoholic fatty liver disease in obese individuals. Am J Clin Nutr 2014;99:869-874. doi: 10.3945/ajcn.113.079749.

[22] Kumar A, Shalimar, Walia GK, Gupta V, Sachdeva MP. Genetics of nonalcoholic fatty liver disease in Asian populations. J Genet 2019;98:29. doi: 10. 1007/s12041-019-1071-8.

[23] Saxena R, Voight BF, Lyssenko V, Burtt NP, de Bakker PI, Chen H, et al. Genome-wide association analysis identifies loci for type 2 diabetes and triglyceride levels. Science 2007;316:1331-1336. doi: 10.1126/science.1142358.

[24] Spars $\varnothing$ T, Andersen G, Nielsen T, Burgdorf KS, Gjesing AP, Nielsen AL, et al. The GCKR rs780094 polymorphism is associated with elevated fasting serum triacylglycerol, reduced fasting and OGTT-related insulinaemia, and reduced risk of type 2 diabetes. Diabetologia 2008;51:70-75. doi: 10.1007/s00125007-0865-z.

[25] Kawaguchi T, Shima T, Mizuno M, Mitsumoto Y, Umemura A, Kanbara Y, et al. Risk estimation model for nonalcoholic fatty liver disease in the Japanese 
using multiple genetic markers. PLoS One 2018;13:e0185490. doi: 10 1371/journal.pone.0185490.

[26] Petit JM, Masson D, Guiu B, Rollot F, Duvillard L, Bouillet B, et al. GCKR polymorphism influences liver fat content in patients with type 2 diabetes. Acta Diabetol 2016;53:237-242. doi: 10.1007/s00592-015-0766-4.

[27] Di Costanzo A, Pacifico L, D'Erasmo L, Polito L, Martino MD, Perla FM, et al. Nonalcoholic fatty liver disease (NAFLD), but not its susceptibility gene variants, influences the decrease of kidney function in overweight/obese children. Int J Mol Sci 2019;20:E4444. doi: 10.3390/ijms20184444.

[28] Simons PIHG, Simons N, Stehouwer CDA, Schalkwijk CG, Schaper NC, Brouwers MCGJ. Association of common gene variants in glucokinase regulatory protein with cardiorenal disease: A systematic review and meta-analysis. PLoS One 2018;13:e0206174. doi: 10.1371/journal.pone.0206174.

[29] Rickham PP. Human experimentation. Code of ethics of the world medical association. declaration of helsinki. Br Med J 1964;2:177. doi: 10.1136/bmj. 2.5402.177.

[30] Kotronen A, Westerbacka J, Bergholm R, Pietiläinen KH, Yki-Järvinen H. Liver fat in the metabolic syndrome. J Clin Endocrinol Metab 2007;92:3490-3497. doi: 10.1210/jc.2007-0482.

[31] Xu Y, Lu LL, Liu SS, Du SX, Zhu HL, Dong QJ, et al. Apolipoprotein A5 gene polymorphisms are associated with non-alcoholic fatty liver disease. Hepatobiliary Pancreat Dis Int 2018;17:214-219. doi: 10.1016/j.hbpd.2018.04.004.

[32] Flores YN, Velázquez-Cruz R, Ramírez P, Bañuelos M, Zhang ZF, Yee HF Jr, et al. Association between PNPLA3 (rs738409), LYPLAL1 (rs12137855), PPP1R3B (rs4240624), GCKR (rs780094), and elevated transaminase levels in overweight/obese Mexican adults. Mol Biol Rep 2016;43:13591369. doi: 10.1007/s11033-016-4058-z.

[33] Kitamoto A, Kitamoto T, Nakamura T, Ogawa $Y$, Yoneda $M$, Hyogo $H$, et al. Association of polymorphisms in GCKR and TRIB1 with nonalcoholic fatty liver disease and metabolic syndrome traits. Endocr J 2014;61:683-689. doi: 10.1507/endocrj.ej14-0052.
[34] Bamshad M, Kivisild T, Watkins WS, Dixon ME, Ricker CE, Rao BB, et al. Genetic evidence on the origins of Indian caste populations. Genome Res 2001;11:994-1004. doi: 10.1101/gr.gr-1733rr.

[35] Hayward BE, Dunlop N, Intody S, Leek JP, Markham AF, Warner JP, et al. Organization of the human glucokinase regulator gene GCKR. Genomics 1998;49:137-142. doi: 10.1006/geno.1997.5195.

[36] Grimsby J, Coffey JW, Dvorozniak MT, Magram J, Li G, Matschinsky FM, et al. Characterization of glucokinase regulatory protein-deficient mice. J Biol Chem 2000;275:7826-7831. doi: 10.1074/jbc.275.11.7826.

[37] Beer NL, Tribble ND, McCulloch LJ, Roos C, Johnson PR, Orho-Melander M, et al. The P446L variant in GCKR associated with fasting plasma glucose and triglyceride levels exerts its effect through increased glucokinase activity in liver. Hum Mol Genet 2009;18:4081-4088. doi: 10.1093/hmg/ddp357.

[38] Rees MG, Wincovitch S, Schultz J, Waterstradt R, Beer NL, Baltrusch S, et al. Cellular characterisation of the GCKR P446L variant associated with type 2 diabetes risk. Diabetologia 2012;55:114-122. doi: 10.1007/s00125-0112348-5.

[39] Shen H, Pollin TI, Damcott CM, McLenithan JC, Mitchell BD, Shuldiner AR. Glucokinase regulatory protein gene polymorphism affects postprandial lipemic response in a dietary intervention study. Hum Genet 2009;126: 567-574. doi: 10.1007/s00439-009-0700-3.

[40] Lian J, Guo J, Chen Z, Jiang Q, Ye H, Huang X, et al. Positive association between GCKR rs780093 polymorphism and coronary heart disease in the aged Han Chinese. Dis Markers 2013;35:863-868. doi: 10.1155/2013/215407.

[41] Járomi L, Csöngei V, Polgár N, Szolnoki Z, Maász A, Horvatovich $K$, et al. Functional variants of glucokinase regulatory protein and apolipoprotein A5 genes in ischemic stroke. J Mol Neurosci 2010;41:121-128. doi: 10. 1007/s12031-009-9301-9.

[42] Bi M, Kao WH, Boerwinkle E, Hoogeveen RC, Rasmussen-Torvik LJ, Astor BC, et al. Association of rs780094 in GCKR with metabolic traits and incident diabetes and cardiovascular disease: the ARIC Study. PLoS One 2010;5: e11690. doi: 10.1371/journal.pone.0011690. 